University of Wollongong

Research Online

Faculty of Engineering - Papers (Archive)

Faculty of Engineering and Information

Sciences

November 2001

\title{
Bulk stress due to surface damage of crystalline silicon and germanium
}

P. Fisher

University of Wollongong, pfisher@uow.edu.au

R. Vickers

University of Wollongong, rv@uow.edu.au

Follow this and additional works at: https://ro.uow.edu.au/engpapers

Part of the Engineering Commons

https://ro.uow.edu.au/engpapers/143

\section{Recommended Citation}

Fisher, P. and Vickers, R.: Bulk stress due to surface damage of crystalline silicon and germanium 2001. https://ro.uow.edu.au/engpapers/143

Research Online is the open access institutional repository for the University of Wollongong. For further information contact the UOW Library: research-pubs@uow.edu.au 


\title{
Bulk stress due to surface damage of crystalline silicon and germanium
}

\author{
P. Fisher ${ }^{\mathrm{a})}$ and R. E. M. Vickers \\ Department of Engineering Physics and Institute for Superconductivity and Electronic Materials, University \\ of Wollongong, Wollongong, New South Wales 2522, Australia
}

(Received 6 August 2001; accepted for publication 4 September 2001)

\begin{abstract}
It is found that abrading two opposing surfaces of either crystalline $\mathrm{Si}$ or Ge samples results in homogeneous, uniaxial stress throughout the bulk perpendicular to the damaged surfaces. The latter are chosen to coincide with simple crystallographic planes. This conclusion is reached by analyzing the splittings, intensities, and polarizations of the absorption lines of the Lyman series of bulk shallow impurities in the abraded semiconductors. This effect has been observed for samples which range in thickness, $t$, from 1 to $3 \mathrm{~mm}$, the internal stress being proportional to $t^{-1}$. (C) 2001 American Institute of Physics. [DOI: 10.1063/1.1418268]
\end{abstract}

The damage produced by surface treatment of semiconductors is of major concern and has been studied extensively over a long period. ${ }^{1-7}$ Much of this work has involved the effect of abrading Si with carborundum. Most of the investigations concentrated on determining the extent of the damage in the surface layer while some, particularly the birefringence studies, ${ }^{2}$ gave an indication of the effect on the bulk. We have studied the effect on the bulk using high resolution far infrared spectroscopy. As will be seen, this has yielded remarkable, unambiguous, and precise information for samples of crystalline $\mathrm{Si}$ and $\mathrm{Ge}$ of up to several millimeters thick.

Bulk samples doped with shallow impurities were prepared for the observation of their Lyman spectra. ${ }^{8}$ These had optical faces coplanar to either $\{100\},\{110\}$, or $\{111\}$; here the optical faces are those on which the infrared radiation falls. The faces remained unpolished after grinding the samples to their desired thickness, $t$. Different grit sizes of $\mathrm{SiC}$ powder were used in a water slurry. The one commonly employed was No. 400, with both faces being treated in the same manner. A sample was mounted stress free in a cryostat at a focus of a Bomem Fourier spectrometer and then immersed in liquid $\mathrm{He}$; this spectrometer provides unpolarized radiation. Band gap radiation was also incident on the sample which decompensated any donors and acceptors contained in the same sample.

The Lyman spectrum of $P$ in $\mathrm{Si}^{8,9}$ is shown, in part, in Fig. 1. The full curve is for a sample with $\{100\}$ optical faces abraded with No. 400 grit SiC; $t=1.91 \mathrm{~mm}$. It had a wedge along its length to suppress Edser-Butler fringes in the region of the $P$ spectrum. The dashed spectrum is from the same sample after etching in a solution of $\mathrm{KOH}$ which reduced $t$ to $1.85 \mathrm{~mm}$. Similar perturbed results were obtained for other values of $t$. The splitting of the $n p_{ \pm}$lines was found to vary linearly as a function of $t^{-1}$ as shown in Fig. 2. For the smaller values of $t$, the wedge was made small enough to give fringes of high visibility in the range of $30-100 \mathrm{~cm}^{-1}$ which permitted the average value of $t$ to be obtained with considerable accuracy. The line through the data points is a least squares fit with a slope of $1.48 \pm 0.02 \mathrm{~cm}^{-1} \mathrm{~mm}$.

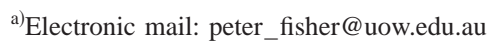

A force, $\mathbf{F}$, applied to crystalline $\mathrm{Si}$ shifts the conduction band minima by

$$
\epsilon_{j}= \pm \Xi_{u}\left(s_{11}-s_{12}\right) S\left(\cos ^{2} \theta_{j}-1 / 3\right), \quad j=1,2, \ldots ., 6,
$$

where $\theta_{j}$ is the angle between $\mathbf{F}$ and the direction in $\mathbf{k}$ space of the $j$ th minimum. ${ }^{8}$ In Eq. (1), $\Xi_{u}$ is the shear deformation potential constant of each of the six equivalent band minima, $s_{11}$ and $s_{12}$ are elastic coefficients of the crystal, while $S$ is the stress. The positive sign is for tension and the negative sign for compression. Thus, for $\mathbf{F} \|[100], \epsilon_{1,2}=-2 \epsilon$ and $\epsilon_{3,4,5,6}=\epsilon \equiv \Xi_{u}\left(s_{11}-s_{12}\right) S / 3$. Here, the conduction band

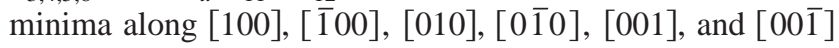
are labeled $1,2,3,4,5$, and 6 , respectively. Also, the electric dipole selection rules for transitions from the singlet ground state, ${ }^{8}$ for $\mathbf{E} \perp \mathbf{F}$, permit transitions to both stress-induced $m$ $= \pm 1$ states but to only the higher energy one of the two stress-induced $m=0$ excited states; here $\mathbf{E}$ is the electric field of the radiation.

The pattern of the spectrum in Fig. 1, from the damaged sample, matches that obtained if an applied, homogeneous compressive force were along a $\langle 100\rangle$ direction with $\mathbf{E} \perp \mathbf{F}{ }^{8,10}$ Thus, it is concluded that the surface damage produces a compressive stress, $S_{\text {eff }}$, throughout the bulk of the sample, perpendicular to the optical faces. Since the radia-

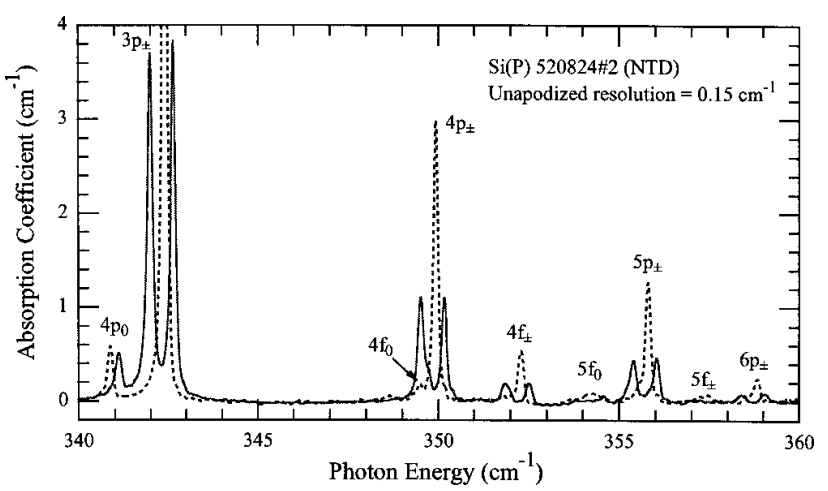

FIG. 1. Part of the Lyman series of $P$ in Si. The full curve is for a sample with $t=1.91 \mathrm{~mm}$ and both optical faces were abraded with No. 400 grit SiC. The dashed spectrum is after the same sample was etched with $\mathrm{KOH}$ to give $t=1.85 \mathrm{~mm}$. The raw spectra were processed to eliminate the effect of the rough surfaces. The concentration of $P$ is $\sim 2 \times 10^{14} \mathrm{~cm}^{-3}$. The labeling of the transitions follows that given in Ref. 8 . 


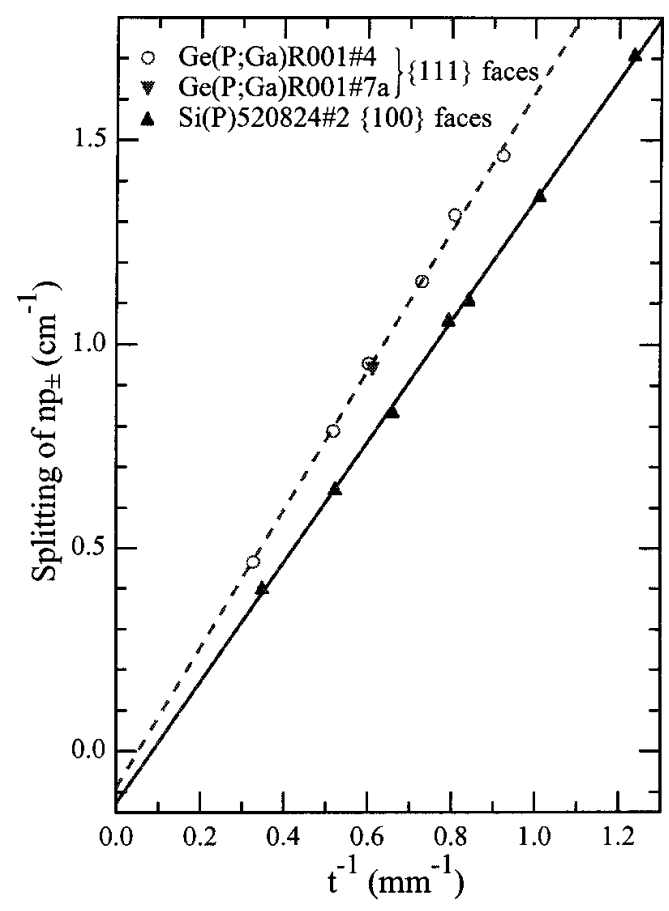

FIG. 2. Dependence on the inverse of the sample thickness of the splitting of the $n p_{ \pm}$states of $P$ in $\mathrm{Si}$ and Ge. The optical faces are $\{100\}$ and $\{111\}$, respectively, and were abraded with No. 400 grit SiC.

tion is at normal incidence, $\mathbf{E}$ will be perpendicular to the stress. From the known values for $\mathrm{Si}$ of $\Xi_{u},{ }^{11} s_{11}$ and $s_{12},{ }^{12}$ and the splitting of the phosphorus $n p_{ \pm}$lines in Fig. $1, S_{\text {eff }}$ is estimated to be $0.94 \pm 0.01 \mathrm{MPa}$.

A phosphorus-doped Si sample with $\{111\}$ optical faces was also examined. No splitting of the $P$ lines was observed, in keeping with the above considerations since, for $\mathbf{F} \|\langle 111\rangle$, Eq. (1) predicts that $\varepsilon_{j}=0$ for all $j$. This is what is obtained experimentally for $\mathbf{F} \|\langle 111\rangle$, applied externally. ${ }^{10}$

The case of $\{110\}$ optical faces for Si has not yet been studied but it has for Ge. Also, for the latter, the $\{111\}$ and $\{100\}$ optical faces have been examined. The shifts of the conduction band minima of $\mathrm{Ge}$ are given by an equation similar to Eq. (1). ${ }^{8}$ For this material there are four equivalent conduction band minima located at the zone boundaries along $\langle 111\rangle$ directions, and, hence, for donors, with $\mathbf{F} \|\langle 100\rangle$ no line splittings should occur while they should for $\mathbf{F} \|\langle 111\rangle$. These characteristics are those observed experimentally when such forces are applied externally, ${ }^{13}$ and are what are seen for spectra obtained from samples of phosphorus-doped Ge with $\{100\}$ and $\{111\}$ optical faces abraded with No. $400 \mathrm{SiC}$ powder in a water slurry. ${ }^{14}$ The dependence on $t^{-1}$ of the splitting of the $n p_{ \pm}$components of $P$ for Ge is shown in Fig. 2 for $\{111\}$ faces. Included in these data are the splittings obtained from two samples cut from the same ingot, illustrating the reproducibility of the process. The line through the data is a least squares fit with a slope of $1.70 \pm 0.04 \mathrm{~cm}^{-1} \mathrm{~mm}$.

From the splitting of the $P$ lines, $S_{\text {eff }}$ is estimated to be $0.92 \pm 0.02 \mathrm{MPa}$ for a sample of Ge with $t=1.92 \mathrm{~mm}$; this is remarkably close to that obtained for the Si sample with $t$ $=1.91 \mathrm{~mm}$ whose spectrum is given in Fig. 1. Unperturbed
Downloaded 15 Aug 2006 to 130.130 .37 .6 . Redistribution subject spectra for Ge could be obtained by subjecting the abraded samples to ultrasonic treatment (UST). The samples were immersed in a beaker of distilled water placed in either a 30 or $43 \mathrm{kHz}$ ultrasonic bath filled with water. During the UST, fine particles collected in the bottom of the beaker and, using a scanning electron microscope, were identified to be Ge. The splitting of the donor states with the time of UST is exponential. ${ }^{14}$ Subsequently, we have learned that $\mathrm{Ge}$ is etched by water. ${ }^{15}$ We found that in this case the reduction in the splitting was very much slower than under UST; also no detritus accumulated.

The phosphorus-doped Ge samples examined contained shallow acceptors. The splittings and intensities ${ }^{16}$ of the stress components of the Lyman spectra of the acceptors confirmed the conclusions deduced from the behavior of the $P$ lines for $\{100\},\{110\}$, and $\{111\}$ optical faces. ${ }^{14}$ Also, it was found that, for a given thickness of Ge, the value of $S_{\text {eff }}$ is essentially the same for all three faces.

The results in Fig. 2 demonstrate that $S_{\text {eff }}$ is proportional to $t^{-1}$. Since the effective strain, $\varepsilon_{\mathrm{eff}}=\Delta t_{\mathrm{eff}} / t$, is proportional to $S_{\text {eff }}$, this implies that $\Delta t_{\text {eff }}$ is constant, independent of $t$. Thus, the surface damage inflicted by abrasion with a grit of a given size leads to a $\Delta t_{\text {eff }}$ which is independent of $t$. It should be noted that $\Delta t_{\text {eff }}$ is not the thickness of the damage inflicted on the surfaces.

Clearly, after abrasion the surfaces are not in equilibrium and, for Ge at least, the elastic energy is released relatively rapidly by UST. Apparently at the power levels and frequencies of the baths used, coupling with acoustic modes in the strained Ge surfaces is sufficient to cause particles to become detached. This extreme reconstruction of the surface did not occur for Si because, it is conjectured, of the stronger covalent bonds which limit the vibrational amplitude of the surface atoms.

The very small, homogeneous stress obtained using the above procedure has provided a better comparison between theoretical and experimental stress-induced effects with more reliable deformation potential constants being obtained ${ }^{14}$ than previously. ${ }^{16}$ This application is being exploited further. Extension of the UST to higher frequencies and power is planned as is the controlled etching of samples to determine the depth of the damage producing the internal stress for correlation with other determinations.

In conclusion, it was shown that the extensive surface damage produced by abrasion of surfaces of crystalline $\mathrm{Si}$ and Ge with carborundum generates amazingly homogeneous, internal stress perpendicular to the damaged surfaces throughout bulk thicknesses of at least $3 \mathrm{~mm}$. This was deduced by analyzing the sharp Lyman spectra of bulk, shallow impurities of samples with and without surface damage. Similar results have also been obtained for $\mathrm{Si}$ and Ge using alumina as the abrading material.

The authors wish to thank D. C. Lau for assistance with the literature survey. The work was supported by the University of Wollongong Board of Research and Postgraduate Studies. The silicon transmutation was carried out under an Australian Institute of Nuclear Science and Engineering grant in conjunction with the Australian Nuclear Science and Technology Organization.

to AIP license or copyright, see http://apl.aip.org/apl/copyright.jsp 
${ }^{1}$ W. C. Dash, J. Appl. Phys. 29, 228 (1958).

${ }^{2}$ W. C. Dash, Phys. Rev. 98, 1536 (1955); S. R. Lederhandler, J. Appl. Phys. 30, 1631 (1959).

${ }^{3}$ B. G. Cohen and M. W. Focht, Solid-State Electron. 13, 105 (1970).

${ }^{4}$ S. Danyluk and R. Reaves, Wear 77, 81 (1982).

${ }^{5}$ S. T. Smith, J. Phys. D 23, 607 (1990).

${ }^{6}$ X-J. Wu, S. Horiuchi, H. Shiwaku, K. Hyodo, and M. Ando, Jpn. J. Appl. Phys., Part 2 31, L803 (1992).

${ }^{7}$ I. Zarudi and L. Zhang, J. Mater. Sci. Lett. 15, 586 (1996); J. Mater. Process. Technol. 84, 149 (1998).

${ }^{8}$ A. K. Ramdas and S. Rodriguez, Rep. Prog. Phys. 44, 1297 (1981).
${ }^{9}$ The phosphorus-doped $\mathrm{Si}$ was obtained by neutron transmutation of essentially undoped $\mathrm{Si}$.

${ }^{10}$ R. L. Aggarwal and A. K. Ramdas, Phys. Rev. A 137, A602 (1965).

${ }^{11}$ V. J. Tekippe, H. R. Chandrasekhar, P. Fisher, and A. K. Ramdas, Phys. Rev. B 6, 2348 (1967). (For Si the value of $\Xi_{u}$ is $8.77 \pm 0.07 \mathrm{eV}$.)

${ }^{12}$ J. J. Hall, Phys. Rev. 161, 756 (1967).

${ }^{13}$ J. H. Reuszer and P. Fisher, Phys. Rev. A 140, A245 (1965).

${ }^{14}$ P. Fisher, B. D. Crowe, R. E. M. Vickers, and A. B. Rosenfeld, Physica B 302/303, 317 (2001).

${ }^{15}$ E. E. Haller (private communication).

${ }^{16}$ A. D. Martin, P. Fisher, C. A. Freeth, E. H. Salib, and P. E. Simmonds, Phys. Lett. 99A, 391 (1983). 